\title{
A new estimation of the lower error bound in balanced truncation method ${ }^{\text {s }}$
}

\author{
Ha Binh Minh ${ }^{\mathrm{a}}$, Carles Batlle ${ }^{\mathrm{b}}$, Enric Fossas ${ }^{\mathrm{c}}$ \\ ${ }^{a}$ School of Applied Mathematics and Informatics, Hanoi University of Science and Technology, 1 Dai Co Viet, Hanoi, Vietnam \\ ${ }^{b}$ Department of Applied Mathematics IV and the Institute of Industrial and Control Engineering, Universitat Politècnica de Catalunya, Spain \\ ${ }^{c}$ Institute of Industrial and Control Engineering, Universitat Politècnica de Catalunya, Spain
}

\begin{abstract}
For a single-input/single-output (SISO) linear time-invariant dynamical system, the classical $\mathcal{H}_{\infty}$-norm lower error bound of balanced truncation method is$$
\left\|G(s)-G_{r}(s)\right\|_{\mathcal{H}_{\infty}} \geq \sigma_{r+1},
$$

where $\sigma_{i}, i=1, \ldots, n$, are the Hankel singular values of system in decreasing order. In this paper we provide a new estimation of the lower error, according to

$$
\left\|G(s)-G_{r}(s)\right\|_{\mathcal{H}_{\infty}} \geq \max \left\{\sigma_{d}, 2\left|\sum_{i \notin \mathcal{J}} s_{i} \sigma_{i}\right|\right\},
$$

where $s_{i}$ is the sign associated with the Hankel singular value $\sigma_{i}$ in Ober's canonical form. The index subset $\mathcal{J}$ and the number $d$ in above inequality will be introduced in the paper. We show by means of an example that the new bound may be relevant in deciding which states to keep in the balanced truncation method, and that using the classical result not always yields the best result.
\end{abstract}

Keywords: linear time-invariant systems, $\mathcal{H}_{\infty}$-norm, lower error bound, balanced truncation, model order reduction, Ober's canonical form.

\section{Introduction}

The $\mathcal{H}_{\infty}$-norm lower and upper error bounds of the balanced truncation method are given by

$$
\sigma_{r+1} \leq\left\|G(s)-G_{r}(s)\right\|_{\mathcal{H}_{\infty}} \leq 2 \sum_{i=r+1}^{n} \sigma_{i},
$$

where $\sigma_{i}, i=1, \ldots, n$, are the Hankel singular values of the system (see e.g., [3, 5]). From these inequalities it follows that, in order to get the smallest error for the truncated system, one should, in any case, disregard the states associated with the smallest Hankel singular values.

Although selecting the truncated system using the above idea yields generally good results, and is widely used in practice, in some cases, as shown in the example at the end of the paper, a smaller error is obtained if one selects differently the states to discard. In this paper we explain this situation by obtaining a better lower error bound, and showing how this may influence the selection of the truncated system.

Our result rests on the new lower bound for the $\mathcal{H}_{\infty}$ norm of a system proposed in $[6,8,11]$, which is based on computing

\footnotetext{
This work was supported by Vietnamese National Foundation for Science and Technology Development (NAFOSTED) under grant 101.02-2013.18, and by Spanish CICYT projects DPI2010-15110 and DPI2011-25649.

Email addresses: minh.habinh@hust. edu.vn;

ha.b.minh@gmail.com (Ha Binh Minh), carles.batlle@upc.edu (Carles Batlle), enric.fossas@upc.edu (Enric Fossas)
}

the transfer function at zero frequency. In this paper, we use the same idea to compute the lower error bound of the balanced truncation method.

Throughout the paper, we denote matrices and vectors by bold-face letters, for example $\mathbf{A}$, and scalars by normal letters, as in $a$. The symbols $\mathbb{R}$ and $\mathbb{C}$ denote the fields of real and complex numbers, respectively.

We consider the class of single-input/single-output (SISO) linear dynamical systems with time-invariant state-space realization

$$
\begin{aligned}
\dot{\mathbf{x}}(t) & =\mathbf{A x}(t)+\mathbf{b} u(t), \\
y(t) & =\mathbf{c x}(t), \quad t \in \mathbb{R},
\end{aligned}
$$

where $(\mathbf{A}, \mathbf{b}, \mathbf{c}) \in \mathbb{R}^{n \times n} \times \mathbb{R}^{n \times 1} \times \mathbb{R}^{1 \times n}, \mathbf{x}(t) \in \mathbb{R}^{n}, u(t) \in \mathbb{R}$ and $y(t) \in \mathbb{R}$. The state-space system (1.2)-(1.3) generates a transfer function

$$
G(s):=\mathbf{c}(s \mathbf{I}-\mathbf{A})^{-1} \mathbf{b}, \quad s \in \mathbb{C}
$$

for which sometimes we use the notation $\left(\begin{array}{c|c}\mathbf{A} & \mathbf{b} \\ \hline \mathbf{c} & \end{array}\right)$.

The $\mathcal{H}_{\infty}$-norm of a linear time-invariant system is defined by

$$
\|G(s)\|_{\mathcal{H}_{\infty}}:=\max _{\omega \in \mathbb{R}} \sigma_{\max }(G(j \omega)),
$$

where $\sigma_{\max }(G(j \omega))$ is the largest singular value of $G(j \omega)$. In the SISO case, however, $G(j \omega)$ is just a complex number, and therefore $\sigma_{\max }(G(j \omega))=|G(j \omega)|$, which gives

$$
\|G(s)\|_{\mathcal{H}_{\infty}}=\max _{\omega \in \mathbb{R}} \sigma_{\max }(G(j \omega))=\max _{\omega \in \mathbb{R}}|G(j \omega)| .
$$


The outline of this paper is as follows. In Section 2 we recall Ober's canonical form of balanced realization [8]. This realization is useful to investigate the $\mathcal{H}_{\infty}$-norm lower error bound of the balanced truncation method, which will be discussed in Section 3. In Section 4, a numerical example is presented, which shows that the classical balanced truncation method does not always yield the best result. Finally, our conclusions are presented in Section 5.

\section{Ober's canonical form of balanced realization}

\subsection{Balanced realization}

Assume that the system $G(s)=\left(\begin{array}{c|c}\mathbf{A} & \mathbf{b} \\ \hline \mathbf{c} & \end{array}\right)$ is asymptotically stable and is in a minimal realization, i.e., $\mathbf{A}$ is stable, the pair $(\mathbf{A}, \mathbf{b})$ is controllable and the pair $(\mathbf{A}, \mathbf{c})$ is observable. The controllability and observability Gramians $\mathbf{P}$ and $\mathbf{Q}$ of the system are, respectively, the solutions of the algebraic Lyapunov equations

$$
\begin{aligned}
& \mathbf{A} \mathbf{P}+\mathbf{P} \mathbf{A}^{T}+\mathbf{b b}^{T}=0 \\
& \mathbf{A}^{T} \mathbf{Q}+\mathbf{Q A}+\mathbf{c}^{T} \mathbf{c}=0 .
\end{aligned}
$$

The balancing transformation is a state transformation that makes the controllability and observability Gramians identical and diagonal, i.e. if the transformation is given by $\mathbf{x}_{b}(t)=$ $\mathbf{T}^{-1} \mathbf{x}(t)$, then

$$
\begin{aligned}
\left(\mathbf{A}_{b}, \mathbf{b}_{b}, \mathbf{c}_{b}\right)=\left(\mathbf{T}^{-1} \mathbf{A T}, \mathbf{T}^{-1} \mathbf{b}, \mathbf{c} \mathbf{T}\right), & \\
\mathbf{P}_{b}=\mathbf{T}^{-1} \mathbf{P} \mathbf{T}^{-T}=\boldsymbol{\Sigma} & :=\operatorname{diag}\left(\sigma_{1}, \sigma_{2}, \ldots, \sigma_{n}\right) \\
& =\mathbf{T}^{T} \mathbf{Q T}=\mathbf{Q}_{b},
\end{aligned}
$$

where $\sigma_{1} \geq \sigma_{2} \geq \cdots \geq \sigma_{n}>0$ are the Hankel singular values of the system. The realization $\left(\mathbf{A}_{b}, \mathbf{b}_{b}, \mathbf{c}_{b}\right)$ is called a balanced realization of system [7].

\subsection{Balanced truncation}

In order to obtain an order reduced model, we assume that $\left(\mathbf{A}_{b}, \mathbf{b}_{b}, \mathbf{c}_{b}\right)$ are in balanced realization. Let $\mathcal{J}:=\left\{i_{1}, \ldots, i_{r}\right\} \subset$ $\{1, \ldots, n\}$ be the indexes of the states that we want to keep in the reduced model. Let $\mathbf{I}_{\mathcal{J}}:=\operatorname{col}\left\{e_{i_{1}}, \ldots, e_{i_{r}}\right\}$, where $e_{j}$ is the $j$-th column vector of identity matrix $\mathbf{I}_{n}$. Then, the reduced-order system $G_{r}(s)$ is obtained by truncating the $(n-r)$ states which do not belong to $\mathcal{J}$, as follows:

$$
\begin{gathered}
\mathbf{A}_{\mathcal{J}}:=\mathbf{I}_{\mathcal{J}} \mathbf{A}_{b} \mathbf{I}_{\mathcal{J}}, \quad \mathbf{b}_{\mathcal{J}}:=\mathbf{I}_{\mathcal{J}} \mathbf{b}_{b}, \quad \mathbf{c}_{\mathcal{J}}:=\mathbf{c}_{b} \mathbf{I}_{\mathcal{J}}, \\
G_{r}(s):=\mathbf{c}_{\mathcal{J}}\left(s \mathbf{I}-\mathbf{A}_{\mathcal{J}}\right)^{-1} \mathbf{b}_{\mathcal{J}} .
\end{gathered}
$$

\subsection{Ober's canonical form of SISO balanced realization}

Suppose that the SISO linear time-invariant system $G(s)=$ $\left(\begin{array}{c|c}\mathbf{A}_{b} & \mathbf{b}_{b} \\ \hline \mathbf{c}_{b} & \end{array}\right)=\mathbf{c}_{b}\left(s \mathbf{I}-\mathbf{A}_{b}\right)^{-1} \mathbf{b}_{b}$ is in balanced realization. Moreover, for simplicity reason, we assume that the Hankel singular values of $G(s)$ are distinct, i.e., $\sigma_{1}>\sigma_{2}>\cdots>\sigma_{n}$. Then in this case, following [8], $G(s)=\left(\begin{array}{c|c}\mathbf{A}_{b} & \mathbf{b}_{b} \\ \hline \mathbf{c}_{b} & \end{array}\right)$ can be written in Ober's canonical form:

$$
G(s)=\left(\begin{array}{cccc|c}
\frac{-b_{1}^{2}}{2 \sigma_{1}} & \frac{-b_{1} b_{2}}{s_{1} s_{2} \sigma_{1}+\sigma_{2}} & \cdots & \frac{-b_{1} b_{n}}{s_{1} s_{n} \sigma_{1}+\sigma_{n}} & b_{1} \\
\frac{-b_{2} b_{1}}{s_{2} s_{1} \sigma_{2}+\sigma_{1}} & \frac{-b_{2}^{2}}{2 \sigma_{2}} & \cdots & \frac{-b_{2} b_{n}}{s_{2} s_{n} \sigma_{2}+\sigma_{n}} & b_{2} \\
\vdots & \vdots & \ddots & \vdots & \vdots \\
\frac{-b_{n} b_{1}}{s_{n} s_{1} \sigma_{n}+\sigma_{1}} & \frac{-b_{n} b_{2}}{s_{n} s_{2} \sigma_{n}+\sigma_{2}} & \cdots & \frac{-b_{n}^{2}}{2 \sigma_{n}} & b_{n} \\
\hline s_{1} b_{1} & s_{2} b_{2} & \cdots & s_{n} b_{n} &
\end{array}\right),
$$

where $s_{i}=1$ or -1 is the sign associated with the Hankel singular value $\sigma_{i}$. Notice that if all the signs $s_{i}$ are equal, either 1 or -1 , then the matrix $\mathbf{A}_{b}$ is symmetric, and $\mathbf{c}_{b}=\mathbf{b}_{b}$ or $\mathbf{c}_{b}=-\mathbf{b}_{b}$. These special cases will be considered later on.

\section{A new lower error bound}

The $\mathcal{H}_{\infty}$-norm of system is always bigger than $|G(0)|$. Moreover, if all the Hankel singular values are distinct then $G(0)$ can be computed, in terms of Hankel's singular values, as follows

$$
G(0)=2 \sum_{i=1}^{n} s_{i} \sigma_{i}
$$

The statement and the proof of this result can be found in [6, $8,11]$. By combining this with the classical lower bound of system [5], we get the following result:

Theorem 3.1. Assume that $G(s)$ is stable SISO system and all the Hankel singular values are distinct. Then,

$$
\|G(s)\|_{\mathcal{H}_{\infty}} \geq \max \left\{\sigma_{1}, 2\left|\sum_{i=1}^{n} s_{i} \sigma_{i}\right|\right\} .
$$

Remark 3.2. One can consider two cases where the new lower bound (3.2) reaches the upper bound of system (see Theorem 4.1 in [10], Theorem 4.1 in [9], or Remark 2.3 in [8]).

(a) In the case that $s_{i}=1$ for all $i=1, \ldots, n$, i.e. the case that $G(s)$ has state-space symmetric realization $\mathbf{A}=$ $\mathbf{A}^{T}, \mathbf{b}=\mathbf{c}^{T}$, then

$$
\|G(s)\|_{\mathcal{H}_{\infty}} \geq 2\left(\sigma_{1}+\cdots+\sigma_{n}\right)
$$

It follows that $\|G(s)\|_{\mathcal{H}_{\infty}}=2\left(\sigma_{1}+\cdots+\sigma_{n}\right)$ since the lower bound is equal to the upper bound.

(b) In the case that $s_{i}=-1$ for all $i=1, \ldots, n$, i.e. the case that $G(s)$ has realization $\mathbf{A}=\mathbf{A}^{T}, \mathbf{b}=-\mathbf{c}^{T}$, then we get the same result as above

$$
\|G(s)\|_{\mathcal{H}_{\infty}}=2\left(\sigma_{1}+\cdots+\sigma_{n}\right)
$$

Now using the same idea as in Theorem 3.1, we are in a position to formulate the main result of this paper.

Theorem 3.3. Assume that $G(s)$ is a stable SISO system and that all the Hankel singular values are distinct. Let $\mathcal{J} \subset\{1, \ldots, n\}$ 
be given, and $d:=\max \{j \mid j \notin \mathcal{J}\}$. If $G_{r}(s)$ is reduced model obtained by the balanced truncation method from $G(s)$, then the lower bound of $\left\|G(s)-G_{r}(s)\right\|_{\mathcal{H}_{\infty}}$ is

$$
\left\|G(s)-G_{r}(s)\right\|_{\mathcal{H}_{\infty}} \geq \max \left\{\sigma_{d}, 2\left|\sum_{i \notin \mathcal{J}} s_{i} \sigma_{i}\right|\right\} .
$$

Proof of Theorem 3.3. Since the balanced truncation method retains the Hankel singular values $\sigma_{i}, i \in \mathcal{J}$, as well as the signs $s_{i}, \in \mathcal{J}$, associated with them, for the reduced system $G_{r}(s)$, we get that

$$
G(0)-G_{r}(0)=2 \sum_{i=1}^{n} s_{i} \sigma_{i}-2 \sum_{i \in \mathcal{J}} s_{i} \sigma_{i}=2 \sum_{i \notin \mathcal{J}} s_{i} \sigma_{i},
$$

which implies that

$$
\begin{aligned}
\left\|G(s)-G_{r}(s)\right\|_{\mathcal{H}_{\infty}} & =\max _{\omega \in \mathbb{R}} \sigma_{\max }\left(G(j \omega)-G_{r}(j \omega)\right) \\
& =\max _{\omega \in \mathbb{R}}\left|G(j \omega)-G_{r}(j \omega)\right| \\
& \geq\left|G(0)-G_{r}(0)\right| \\
& =2\left|\sum_{i \notin \mathcal{J}} s_{i} \sigma_{i}\right| .
\end{aligned}
$$

Now we consider two special cases in Theorem 3.3. In these cases, the upper bound and the lower bound of $\left\|G(s)-G_{r}(s)\right\|_{\mathcal{H}_{\infty}}$ are the same, and therefore the exact error of balanced truncation method can be computed. The proof is omitted since it is obvious.

Corollary 3.4. With the assumptions as in Theorem 3.3, we get that:

(a) In the case that all truncated states have the sign equal to 1 , i.e. $s_{i}=1$ for all $i \notin \mathcal{J}$, the $\mathcal{H}_{\infty}$-norm of $\left(G(s)-G_{r}(s)\right)$ is

$$
\left\|G(s)-G_{r}(s)\right\|_{\mathcal{H}_{\infty}}=2 \sum_{i \notin \mathcal{J}} \sigma_{i}
$$

(b) In the case that all truncated states have the sign equal to -1 , i.e. $s_{i}=-1$ for all $i \notin \mathcal{J}$, we get the same result as above

$$
\left\|G(s)-G_{r}(s)\right\|_{\mathcal{H}_{\infty}}=2 \sum_{i \notin \mathcal{J}} \sigma_{i}
$$

We consider now two special cases, namely when $G(s)$ has state-space symmetric realization $\mathbf{A}=\mathbf{A}^{T}, \mathbf{b}=\mathbf{c}^{T}$, or when $\mathbf{A}=\mathbf{A}^{T}, \mathbf{b}=-\mathbf{c}^{T}$.

Corollary 3.5. With the same assumptions as in Theorem 3.3, one has that

(a) (Theorem 4.4, [9]) In the case that $G(s)$ has state-space symmetric realization $\mathbf{A}=\mathbf{A}^{T}, \mathbf{b}=\mathbf{c}^{T}$, one has

$$
\left\|G(s)-G_{r}(s)\right\|_{\mathcal{H}_{\infty}}=2\left(\sigma_{r+1}+\cdots+\sigma_{n}\right) .
$$

(b) In the case that $G(s)$ has realization $\mathbf{A}=\mathbf{A}^{T}, \mathbf{b}=-\mathbf{c}^{T}$, one gets also

$$
\left\|G(s)-G_{r}(s)\right\|_{\mathcal{H}_{\infty}}=2\left(\sigma_{r+1}+\cdots+\sigma_{n}\right) .
$$

Proof. The results in Corollary 3.5 correspond to the special cases in Corollary 3.4 since all truncated states have the sign 1 or -1 .

\section{A numerical example}

In classical balanced truncation method one truncates the states having the smallest Hankel singular values. The example that we present shows that taking into account the new tighter lower bound may reduce the error on the reduced order model system without increasing its order.

Consider the following linear system, given by Ober's realization form by

$$
\begin{aligned}
\mathbf{b} & =\left[\begin{array}{l}
1 \\
1 \\
1 \\
1 \\
1
\end{array}\right], \quad s=\left[\begin{array}{r}
1 \\
1 \\
-1 \\
1 \\
1
\end{array}\right], \quad \sigma=\left[\begin{array}{c}
5 \\
4 \\
3 \\
2.5 \\
2
\end{array}\right], \\
\mathbf{c} & =\left[\begin{array}{lllll}
s_{1} b_{1} & s_{2} b_{2} & \cdots & s_{n} b_{n}
\end{array}\right], \\
& =\left[\begin{array}{lllll}
1 & 1 & -1 & 1 & 1
\end{array}\right],
\end{aligned}
$$

$$
\begin{aligned}
\mathbf{A} & =\left[\begin{array}{cccc}
\frac{-b_{1}^{2}}{2 \sigma_{1}} & \frac{-b_{1} b_{2}}{s_{1} s_{2} \sigma_{1}+\sigma_{2}} & \cdots & \frac{-b_{1} b_{n}}{s_{1} s_{n} \sigma_{1}+\sigma_{n}} \\
\frac{-b_{2} b_{1}}{s_{2} s_{1} \sigma_{2}+\sigma_{1}} & \frac{-b_{2}^{2}}{2 \sigma_{2}} & \cdots & \frac{-b_{2} b_{n}}{s_{2} s_{n} \sigma_{2}+\sigma_{n}} \\
\vdots & \vdots & \ddots & \vdots \\
\frac{-b_{n} b_{1}}{s_{n} s_{1} \sigma_{n}+\sigma_{1}} & \frac{-b_{n} b_{2}}{s_{n} s_{2} \sigma_{n}+\sigma_{2}} & \cdots & \frac{-b_{n}^{2}}{2 \sigma_{n}}
\end{array}\right] \\
& =\left[\begin{array}{ccccc}
-0.100 & -0.111 & 0.500 & -0.133 & -0.143 \\
-0.111 & -0.125 & 1.000 & -0.154 & -0.167 \\
-0.500 & -1.000 & -0.167 & 2.000 & 1.000 \\
-0.133 & -0.154 & -2.000 & -0.200 & -0.222 \\
-0.143 & -0.167 & -1.000 & -0.222 & -0.250
\end{array}\right] .
\end{aligned}
$$

Let $G(s)=\left(\begin{array}{c|c}\mathbf{A} & \mathbf{b} \\ \hline \mathbf{c} & \end{array}\right) . G(s)$ is in balanced realization and its Hankel singular values are

$$
\sigma_{1}=5, \sigma_{2}=4, \sigma_{3}=3, \sigma_{4}=2.5, \sigma_{5}=2
$$

If we truncate the 2 states having the smallest Hankel singular values, $\sigma_{4}$ and $\sigma_{5}$, as in the classical balanced truncation method, we obtain the following reduced-order system.

$$
G_{1}(s)=\left(\begin{array}{ccc|c}
-0.100 & -0.111 & 0.500 & 1 \\
-0.111 & -0.125 & 1.000 & 1 \\
-0.500 & -1.000 & -0.167 & 1 \\
\hline 1 & 1 & -1 &
\end{array}\right) .
$$

The $\mathcal{H}_{\infty}$-norm of error in this case is

$$
\left\|G(s)-G_{1}(s)\right\|_{\mathcal{H}_{\infty}}=9
$$

which is attained at frequency $\omega=0$.

However, if we truncate the 2 states corresponding to $\sigma_{2}$ and $\sigma_{3}$ we obtain the following reduced-order system.

$$
G_{2}(s)=\left(\begin{array}{ccc|c}
-0.100 & -0.133 & -0.143 & 1 \\
-0.133 & -0.200 & -0.222 & 1 \\
-0.143 & -0.222 & -0.250 & 1 \\
\hline 1 & 1 & 1 &
\end{array}\right) \text {. }
$$


The $\mathcal{H}_{\infty}$-norm of error in this case is

$$
\left\|G(s)-G_{2}(s)\right\|_{\mathcal{H}_{\infty}}=5.6421,
$$

which is now attained at frequency $\omega=2.4814$.

Hence, truncating the states having the smallest Hankel singular values does not always give the best reduced system. One can find an explanation of this result in the light of the new lower error bound given in Theorem 3.3. One has that

$$
2\left(\sigma_{4}+\sigma_{5}\right) \geq\left\|G(s)-G_{1}(s)\right\|_{\mathcal{H}_{\infty}} \geq \max \left\{\sigma_{4}, 2\left(\sigma_{4}+\sigma_{5}\right)\right\},
$$

and, therefore, $9 \geq\left\|G(s)-G_{1}(s)\right\|_{\mathcal{H}_{\infty}} \geq 9$, or $\left\|G(s)-G_{1}(s)\right\|_{\mathcal{H}_{\infty}}=$ 9. On the other hand,

$$
2\left(\sigma_{2}+\sigma_{3}\right) \geq\left\|G(s)-G_{2}(s)\right\|_{\mathcal{H}_{\infty}} \geq \max \left\{\sigma_{2}, 2\left|\sigma_{2}-\sigma_{3}\right|\right\}
$$

which leads to $14 \geq\left\|G(s)-G_{2}(s)\right\|_{\mathcal{H}_{\infty}} \geq 4$. The lower bound of $\left\|G(s)-G_{2}(s)\right\|_{\mathcal{H}_{\infty}}$ is smaller than the one of $\left\|G(s)-G_{1}(s)\right\|_{\mathcal{H}_{\infty}}$, so it may yield a better result, and in fact it does in our example.

\section{Conclusions}

This paper has shown that the $\mathcal{H}_{\infty}$-norm lower bound of SISO linear systems as well as the $\mathcal{H}_{\infty}$-norm lower error bound of balanced truncation method can be improved. The technique is based on the computation of transfer function at zero frequency. The key point in this paper is the balanced realization obtained by Ober [8] for the SISO linear systems. Whether the new bound improves the classical one depends on the detailed numerical values of the Hankel singular values of the system, as well as of their signs, but we have shown an explicit example where the new bound is relevant.

We should comment that these results can not be extended to the case of multi-input/multi-output (MIMO) linear timeinvariant systems. This is due to the fact that equality (3.1) does not hold for the MIMO case. The question of defining a systematic way to improve the balanced truncation given the spectrum of Hankel's singular values and their associated signs is an open one.

[1] A.C. Antoulas, Approximation of Large-Scale Dynamical Systems, SIAM Press, 2005.

[2] Robert R. Bitmead and Brian D. O. Anderson, The Matrix Cauchy Index: Properties and Applications, SIAM J. Appl. Math., 33(4) (1977), pp. 655-672.

[3] D.F. Enns, Model reduction with balanced realizations: An error bound and a frequency weighted generalization, Proceedings of the $23^{r d}$ Control and Decision Conference (Las Vegas), (1984), pp. $127-132$.

[4] Ha Binh Minh, Model reduction in a behavioral framework, Doctoral Dissertation University of Groningen, 2009. pp. 1-10.

[5] K. Glover, All optimal Hankel-norm approximations of linear multivariable systems and their $L^{\infty}$-error bounds, Internat. J. Control, 39(4) (1984), pp. 1115-1193.

[6] S.S. Mahil, F.W. Faiman, B.S. Lee, Some integral properties for balanced realizations of scalar systems, IEEE Trans. Automat. Control, 29 (1984), pp. 181-183.

[7] B.C. Moore, Principal component analysis in linear systems: controllability, observability, and model reduction, IEEE Trans. Automat. Control, 26(2) (1981), pp. 17-32.

[8] R. Ober, Balanced Parametrization of Classes of Linear Systems, SIAM J. Control Optim., 29 (1991), pp. 1251-1287.
[9] Timo Reis, Tatjana Stykel, Lyapunov Balancing for PassivityPreserving Model Reduction of RC Circuits, SIAM J. Applied Dynamical Systems, 10 (2011), No. 1, pp. 1-34.

[10] W.Q. Liu, V. Sreeram, K.L. Teo, Model reduction for state-space symmetric systems, Systems Control Lett., 34 (1988), pp. 209-215.

[11] D.A. Wilson, A. Kumar, Symmetry properties of balanced systems, IEEE Trans. Automat. Control, 28 (1983), pp. 927-929. 